\title{
Factores que inciden en la calidad de vida de las personas adultas mayores ${ }^{1}$
}

\author{
Institución: Universidad de Costa Rica \\ CÓMO CITAR
}

\author{
Mabel Granados Hernández ${ }^{2}$ \\ Derby Muñoz Rojas ${ }^{3}$
}

\begin{abstract}
Granados, M. y Muñoz, D. (2015). Factores que inciden en la calidad de vida de las personas adultas mayores. Rev. Enfermería Actual en Costa Rica, 29, 1-16. DOI: http://dx.doi.org/10.15517/revenf.v0i29.19732
\end{abstract}

\section{RESUMEN}

Introducción. El objetivo de este artículo es presentar los resultados del proyecto de investigación $\mathrm{N}^{\mathrm{o}}$.421-B1234 de la Escuela de Enfermería de la Universidad de Costa Rica, Práctica de enfermería basada en la evidencia para mejorar la calidad de vida de las personas adultas mayores, el cual pertenece al proyecto $\mathrm{N}^{\mathrm{o}}$. 421-B1-910 Programa para la Colaboración para la Investigación de Enfermería Basada en la Evidencia de Costa Rica. En este escrito se presenta una revisión teórica de los trabajos más importantes respecto de dicha temática, con el objetivo de aclarar qué factores y variables son los que inciden en tener una mayor calidad de vida en la actualidad en las personas adultas mayores.

Método. La metodología utilizada es la que se recomienda para desarrollar la práctica clínica basada en la evidencia con sus respectivas etapas. En cuanto a los documentos, inicialmente se recuperó un total de 504 de diferentes bases de datos incluyendo PUBMED, EBSCO, LILACS, CINAHL, y COCHRANE, de los cuales únicamente 30 fueron retenidos ya que incluían revisiones de literatura, estudios primarios, y marcos conceptuales relevantes para la pregunta clínica. Finalmente, por su contenido, seis se emplearon para el análisis y la lectura crítica por su estructura metodológica, y calidad, dado que fundamentan indirectamente la respuesta a la pregunta clínica que guió el presente estudio.

Resultados. Se evidencia que la musicoterapia puede tener un efecto positivo en la calidad de vida de las personas adultas mayores, la cual puede ser influida por factores individuales, sociales, culturales, y ambientales, el uso de dispositivos electrónicos, la conectividad social, la respuesta adaptativa y la interacción positiva con otras personas. Por último, las implicaciones para la investigación, práctica de enfermería, política, y conocimiento enfermero son discutidos de manera detallada.

Conclusión. Existe necesidad de desarrollar más estudios sobre los factores que intervienen en la calidad de vida de las personas adultas mayores, a través del metaanálisis o de revisiones sistemáticas, que permitirían corregir el vacío de información en este campo.

Descriptores: calidad-de-vida, enfermería-basada-en-la-evidencia, práctica-de-enfermería, persona-adulta-mayor

\footnotetext{
${ }^{1}$ Fecha de recepción: 5 de mayo del 2015

Fecha de aceptación: 22 de junio del 2015

${ }^{2}$ Enfermera. Profesora Escuela de Enfermería de la Universidad de Costa Rica. Costa Rica. E-mail: mabelmgranados@gmail.com

${ }^{3}$ Enfermero. Profesor Escuela de Enfermería de la Universidad de Costa Rica. Costa Rica. E-mail: derby.munoz@ucr.ac.cr derbymr@gmail.com
} 


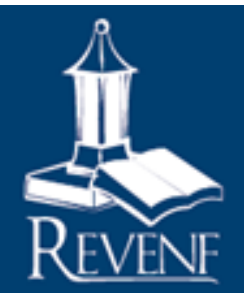

\title{
Revista Electrónica Entermeria actual en costa Rica
}

\section{Factors affecting the quality of life of older people ${ }^{1}$}

\author{
Institución: Universidad de Costa Rica
}

\author{
Mabel Granados Hernández ${ }^{2}$ \\ Derby Muñoz Rojas ${ }^{3}$
}

\section{CÓMO CITAR}

Granados, M. y Muñoz, D. (2015). Factors affecting the quality of life of older people. Rev. Enfermería Actual en Costa Rica, 29, 1-16. DOI: http://dx.doi.org/10.15517/revenf.v0i29.19732

\begin{abstract}
Introduction. The aim of this article is to present the results of the research project $\mathrm{N}^{0}$. 421-B1-234 School of Nursing at the University of Costa Rica, known as Evidence-based nursing practice to improve the quality of life of the elderly, which belongs to the project $\mathrm{N}^{0}$. 421-B1-910 Program for Collaborative Evidence-Based Nursing Research of Costa Rica. This research is presented as a theoretical review of the most important studies in relation to this issue, in order to clarify what type of factors and variables influence the quality of life in the elderly.

Method. The methodology used is the one recommended to develop clinical practice based on evidence with their respective stages. A total of 504 papers were initially retrieved from different databases, including PubMed, EBSCO, LILACS, CINAHL, and COCHRANE. Of these documents, only 30 were retained since they included literature reviews, primary studies, and conceptual frameworks relevant to the clinical question. Finally, six of these documents were retained for the analysis and critical reading due to its content,_methodological structure, and quality, which indirectly answer the clinical question that guided this study.

Result. Findings show that music therapy can have a positive effect on the quality of life of older people. Also, quality of life may also be positively influenced by individual, social, cultural, and environmental factors, such as use of electronic devices, social connectivity, adaptive response, and positive interaction with others. Implications for nursing research, practice, policy, and knowledge are discussed in detail.

Conclusion. There is need for further studies on the factors involved in the quality of life of older people through the meta-analysis or systematic reviews, which allow to correct the information gap in this field.
\end{abstract}

Key words: elderly, evidence-based-nursing, quality-of-life, nursing-practice

\footnotetext{
${ }^{1}$ Date of receipt: May 5, 2015

Date of acceptance: June 22, 2015

${ }^{2}$ Enfermera. Profesora Escuela de Enfermería de la Universidad de Costa Rica. Costa Rica. E-mail: mabelmgranados@gmail.com

${ }^{3}$ Enfermero. Profesor Escuela de Enfermería de la Universidad de Costa Rica. Costa Rica. E-mail: derby.munoz@ucr.ac.cr derbymr@gmail.com
} 


\section{Revista Electrónica Enfermeria Actual en costa Rica}

\section{INTRODUCCIÓN}

El objetivo de este artículo es presentar los resultados del proyecto de investigación $\mathrm{N}^{0}$. 421-B1-234, Práctica de enfermería basada en la evidencia para el mejoramiento de la calidad de vida de las personas adultas mayores, inscrito en la Vicerrectoría de Investigación de la Universidad de Costa Rica.

Actualmente, los esfuerzos de los profesionales de enfermería se enfocan en la consolidación de un cuerpo de conocimientos concretos para el continuo desarrollo de la enfermería como disciplina científica y profesión reconocida; para lograrlo, es fundamental asegurar una práctica basada en la evidencia respaldada por el avance de la investigación, tanto en el campo clínico y comunitario, como en la gestión y la educación. En enfermería, la práctica, la disciplina y la investigación están íntimamente relacionadas: el conocimiento de la disciplina proviene de la relación recíproca entre la teoría, el conocimiento y la práctica, vínculo que permite a la profesión enfrentar de mejor manera sus obligaciones con la sociedad y por ello el conocimiento producto de la investigación en enfermería debe ser accesible, relevante y significativo para la sociedad, siempre teniendo claro el objeto de estudio de la profesión, el cuidado, visto como un servicio susceptible de mejorarse permanentemente, mediante estrategias de mejoramiento continuo del cuidado.

Dicha mejora incluye la enfermería basada en la evidencia la cual se construye sobre una base de elementos como los resultados de la revisión bibliográfica, el consenso profesional y la experiencia sistematizada de profesionales del área conocedores de la metodología, quienes trabajan en lo que debe hacerse, para quien deba hacerse y de la mejor manera posible, al tiempo que mejoran la efectividad y la eficiencia de los cuidados de enfermería (Ochoa, 2005, p.140). De igual manera, dicho método permite generar conocimientos, mejorar la educación y la práctica profesional y utilizar efectivamente los recursos disponibles para beneficiar el objeto de estudio: el cuidado de los individuos y colectivos humanos y la calidad de vida.

La enfermería basada en la evidencia se propone como un modelo en el cual se plantea preguntas de investigación procedentes de los problemas cotidianos de la práctica, cuya respuesta se evalúa sistemáticamente a partir de resultados de investigación rigurosa que oriente las mejores decisiones en beneficio de la persona usuaria, lo cual significa que las actuaciones de los profesionales de enfermería no quedan relegados a la experiencia personal, a la intuición, al sentido común, a la tradición o a normas preestablecidas, sino que son respaldadas por el rigor metodológico a partir de la evidencia científica disponible, siempre y cuando ofrezca resultados aplicables y pertinentes que mejoren la calidad de la prestación del servicio de enfermería y los servicios de salud, incremente la satisfacción de los usuarios y viabilice la formulación de políticas en salud y de la profesión de enfermería (Ochoa, 2005). A lo anterior, cabe agregar que se perfila como un instrumento válido para sustentar la práctica de enfermería y mejorar la calidad de atención, razón por la que una práctica basada en la evidencia es necesaria para proporcionar cuidados de salud en forma equitativa y efectiva para toda la población (Urra, Retamal, Tapia \& Rodríguez, 2010), en este caso de las personas adultas mayores.

El siglo XXI se caracteriza por estar compuesto por una población con tendencia a envejecer y una sociedad en cuya estructura se consolida la abundante presencia de personas adultas mayores, motivo por el que se ha de buscar estrategias para la mejora de la calidad de vida de dicho sector, definida por la Organización Mundial de la Salud (1994), como "la percepción del individuo sobre su posición en la vida dentro del contexto cultural y el sistema de valores en el que vive y con respecto a sus metas, expectativas, normas y preocupaciones" (p. 49): se trata de un concepto extenso y complejo que engloba la salud física, el proceso psicológico, el nivel de 
independencia, las relaciones sociales, las creencias personales y la relación con las características sobresalientes del entorno.

Dado lo anterior, este trabajo se presenta como una revisión teórica de los trabajos más importantes relacionados con la temática, cuyo objetivo es aclarar qué factores y variables propician una mayor calidad de vida en la actualidad en las personas adultas mayores. Para llevarlo a cabo se planteó la siguiente pregunta utilizando para ello el acrónimo P (población) I (intervención) C (comparación) O (resultados): ¿Cuál práctica de enfermería basada en la evidencia mejora la calidad de vida de las personas adultas mayores?

\section{MATERIALES Y MÉTODOS}

En cuanto a la metodología, se emplea la recomendada para desarrollar la práctica clínica basada en la evidencia (Rojas, Cubero y Leiva, 2014) la cual consta de un método de cinco etapas, con lo que se pretende que, al concluir el círculo de la EBE, se constituya en un proceso de mejora de la calidad asistencial y de garantía de una práctica segura.

\section{Primera etapa: La elaboración de preguntas clínicas como paso inicial de la práctica de la EBE}

En la práctica diaria la necesidad de información para la toma de decisiones es un aspecto fundamental; no obstante, suele darse el problema de la "excesiva información", escenario en el que es imprescindible formular correctamente nuevas dudas clínicas en forma de preguntas y utilizar fuentes de información sencillas y eficientes. La formulación de la pregunta se deriva del problema observado y surge de variadas fuentes, ya sea la curiosidad intelectual o de la necesidad de responder a preguntas sobre un tema clínico.

\section{Segunda etapa: Búsqueda de la información}

Para cumplir esta etapa, se debe buscar la mejor respuesta a la pregunta en las fuentes de información que, según Mckibbon (1998), en Galváo et al., (2003) y Lopes Monteiro y de Mattos (2005) serían los libros y textos, llamados también conocimiento estable, publicaciones periódicas, bases bibliográficas electrónicas, bases de información específicas de EBE y, por último, internet, cuyo uso demanda un análisis de la calidad de los sitios visitados y de la información contenida en ellos. De igual forma, Icart (2000) incluye las guías de práctica clínica que proporcionan recomendaciones a los profesionales y usuarios para tomar decisiones de intervención según la condición clínica presentada. La necesidad de recolectar las mejores evidencias hace de la revisión de la literatura un elemento muy importante en el proceso, por lo que las bases de datos en enfermería y salud son esenciales.

\section{Tercera etapa: Lectura crítica}

La evaluación crítica de la evidencia encontrada consiste en el proceso de evaluar e interpretar la evidencia aportada por la bibliografía científica, considerando sistemáticamente los resultados que se presentan, su validez y su relevancia para el propio trabajo. En este mismo sentido, Gómez de la Cámara (1998) sostiene que el uso óptimo de la información científica contrastada y fiable, procedente de la mejor investigación, sería la clave para 
mejorar la eficacia de la práctica clínica y la atención sanitaria en general, al reducir la incertidumbre y aumentar la estima y el aval ético del profesional.

La lectura crítica se compone de tres pasos mediante los que se determina los diferentes niveles de análisis de los artículos y se emplea el tiempo de forma correcta, ya que facilita la labor de eliminación de artículos que no cumplen los criterios de calidad científica buscados. En el primer paso, se puede descartar artículos que no se centren en el tema; en el segundo se analiza la capacidad del artículo para contestar al problema planteado y en el tercero, el análisis de los artículos que hayan superado los dos pasos anteriores es más profundo, dado que se enfoca la evaluación sobre el material y la metodología utilizados

\section{Cuarta etapa: Implementación de la evidencia}

Se implementa las evidencias en la práctica, con el objetivo de mejorar la calidad de los cuidados entregados a las personas y a sus familias de modo que, según Castrillón (2001), el acto del cuidado sea una actividad reflexiva, deliberada y autónoma que considere las preferencias de la o las personas a quienes va dirigido. En esta fase, se debe contar con profesionales de enfermería que utilicen en la práctica diaria las evidencias obtenidas a través de la investigación, una tarea difícil, debido a que es un largo camino el que debe realizarse a través de la formación y estímulo tanto de las enfermeras interesadas como de las futuras generaciones.

\section{Quinta etapa: Evaluación}

En la última etapa se evalúa las consecuencias de aplicar la intervención elegida, razón por la que se destaca su importancia, dado que permite la retroalimentación permanente entre la investigación y la clínica. Por otra parte, se revisa las consecuencias de los cambios introducidos en la práctica, para identificar si han sido o no efectivos, a lo que se añade que se debe centrarse en los resultados, más que en el proceso.

La estrategia de búsqueda para identificar los estudios usados en la investigación se basó en los siguientes conceptos: enfermería basada en la evidencia, calidad de vida, personas adultas mayores, práctica de enfermería e investigación en enfermería. Los criterios de inclusión para la búsqueda se basaron en documentos del año 2005 al 2013, en idioma español e inglés. Los descriptores o las palabras clave utilizados fueron persona adulta mayor, mejoramiento calidad de vida, práctica de enfermería, enfermería basada en evidencia, vejez.

Se realizó el levantamiento bibliográfico de publicaciones indexadas o catalogadas en las bases de datos PUBMED, Ebsco, LILACS, CINAHL, COCHRANE, National Guideline Clearing House (NGC) y Scielo

En la delimitación de la búsqueda se consideró el mejoramiento de la calidad de vida en personas adultas mayores. El levantamiento corresponde al periodo 2012-2014 y la primera selección de los artículos mediante el análisis de los resúmenes de 504 documentos. Luego, se empleó límites de búsqueda por fecha del 2005-2014 y se obtuvo 334 documentos que, tras el filtro por título y resumen, se obtuvo 296; por último, incluyendo tan solo 30 documentos por texto completo por título y resumen, los cuales corresponden a revisiones de literatura, estudios primarios y marcos conceptuales relevantes para dar respuesta a la pregunta clínica. De los 30 documentos, solo 
se consideró seis estudios por su contenido, estructura metodológica y calidad. A pesar de que directamente no respondieron a la pregunta clínica, sus contenidos responden a la temática investigada en forma indirecta, lo que permitió puntualizar el grado de recomendación y el nivel de evidencia.

\section{Instrumentos}

Lectura crítica: la evidencia aportada por los artículos seleccionados fue evaluada utilizando dos sistemas diferentes según el método de investigación descrito en el artículo correspondiente.

Métodos cuantitativos: Las fichas de lectura crítica (FLC 2.0) fueron desarrolladas por Osteba, Servicio de Evaluación de Tecnologías Sanitarias del Departamento de Salud del Gobierno Vasco (López et al., 2006). FLC 2.0 es una aplicación web diseñada para apoyar el desarrollo de revisiones sistemáticas de la evidencia científica, al punto de que son un apoyo a la evaluación de la calidad de los estudios científicos y a la síntesis de la evidencia. Estas plantillas permiten evaluar la pregunta de investigación, el método, los resultados, las conclusiones, la existencia de conflictos de interés, y la validez interna por medio de preguntas abiertas y cerradas que sintetizan la información específica de cada sección. Un ejemplo de una pregunta es ¿los autores declaran la existencia o ausencia de algún conflicto de interés? Con base en la evidencia provista para cada sección, esta es evaluada con en una escala de cuatro valores (i.e., Bien, Regular, Mal, Ns/No Aplica) y luego, se clasifica la evidencia usando una escala de tres valores (i.e., Baja, Media, y Alta).

Métodos cualitativos: Las Guidelines for Critical Review Form: Qualitative Studies (Version 2.0) fueron desarrolladas por McMaster University Occupational Therapy Evidence-Based Practice Research Group (Letts et al., 2007). Estas guías, en este caso en formato de papel y lápiz, permiten evaluar críticamente la evidencia aportada por cada artículo, lo cual incluye la cita de referencia, el propósito, la revisión de la literatura, el diseño y su pertinencia, los métodos utilizados, el muestreo, la recolección de datos, la auditoría de calidad, la rigurosidad y las conclusiones e implicaciones, a través de preguntas abiertas y cerradas que sintetizan la información específica de cada sección. Un ejemplo de una pregunta es ¿fue el consentimiento informado obtenido?

Nivel de recomendación: Los grados recomendación establecidos por la Canadian Task Force on Preventive Health Care (2014) fueron utilizados para establecer el nivel de la evidencia evaluada. Estos grados van en un rango de seis niveles que van de $A$ hasta $I$, en los que A es el máximo nivel posible (es decir, hay buena evidencia para recomendar la acción clínica preventiva), mientras que grado $I$ es el inferior (que no hay suficiente evidencia para hacer una recomendación, sin embargo otros factores podrían influenciar la decisión). La categorización en estos niveles depende de los siguiente seis criterios: el problema, los beneficios y riesgos de la intervención, el uso de recursos, la equidad, la aceptabilidad, y la factibilidad (Canadian Task Force on Preventive Health Care, 2014).

\section{Consideraciones éticas}

Se tomó en cuenta que cada artículo cumpliera con una estructura ética en el manejo de las personas participantes, así como con el uso de la información. A la vez se guardó rigurosamente la identidad de los autores y los títulos de los diferentes trabajos de investigación usados en el análisis. Además, los procedimientos de 
investigación de este estudio fueron aprobados por la Vicerrectoría de Investigación de la Universidad de Costa Rica.

\section{RESULTADOS}

Durante la fase de búsqueda de la evidencia se recuperó seis artículos, los cuales indirectamente fundamentan la respuesta a la pregunta clínica que guió el presente estudio: ¿Cuál práctica de enfermería basada en la evidencia mejora la calidad de vida de las personas adultas mayores? A continuación se describen los artículos, así como los resultados y conclusiones pertinentes de esta investigación.

Chan, S., Shoumei, J., Thompson, D., Yan, H., Chiu, H., Chien, W. \& Lam, L. (2006). A cross-sectional study on the health related quality of life of depressed Chinese older people in Shanghai. International Journal of Geriatric Psychiatry, 21(9), 883-889. doi:10.1002/gps.1578

Esta investigación tuvo como objetivos a) investigar la autopercepción sobre la calidad de vida de personas adultas mayores diagnosticadas con depresión viviendo en una comunidad en Shanghái, y b) examinar la relación entre la calidad de vida y la salud física y mental, el apoyo social, y el estado funcional. El trabajo realizado en el año 2005, correspondió a un diseño transversal en el que los investigadores aplicaron un cuestionario a 71 personas adultas mayores con depresión. El instrumento incluyó preguntas sobre percepción subjetiva de la calidad de vida relacionada con la salud, nivel de depresión, funcionamiento cognitivo, condiciones médicas, actividades diarias, habilidades funcionales, y nivel de apoyo social. Los resultados indican que un alto grado de depresión es significativamente asociado a una percepción negativa de la calidad de vida ligada a la salud. Los participantes reportaron estar poco satisfechos con su estado de salud física. El nivel de depresión $(\beta=-0.72, p<0.001)$, de actividades diarias $(\beta=0.17, p<0.001)$, y de satisfacción con el apoyo social $(\beta=0.12, p<0.001)$ fueron factores significativamente predictivos del grado de percepción de la calidad de vida ligada a la salud.

Los autores concluyeron que el estudio identificó cómo la depresión afecta el estado biopsicosocial de las personas adultas mayores en China. Los resultados fueron discutidos considerando el contexto sociocultural de Shanghái.

Respecto de la lectura crítica de este artículo, en términos de la calidad de la evidencia se encontró que el estudio se basa en una pregunta de investigación que es regularmente definida. La metodología empleada garantiza una validez interna regular del estudio. Los resultados del estudio están bien descritos. Las conclusiones presentadas están bien basadas en los resultados obtenidos y consideran las posibles limitaciones del estudio. No hay información acerca de los conflictos de interés por parte de los investigadores. Por último, los resultados del estudio pueden ser bien generalizados a la población y al contexto que interesa; por ende, se considera que la calidad de la evidencia es media.

Hawton, A., Green, C., Dickens, A., Richards, S., Taylor, R., Edwards, R., Greaves, C., \& Campbell, J. (2011). The impact of social isolation on the health status and health-related quality of life of older people. Qual Life Res, 20:57-67. doi 10.1007/s11136-010-9717-2 
Los objetivos de este estudio consistieron en investigar la relación entre a) el estado de salud y la calidad de vida relacionada con la salud (CVRS) y b) el abandono social y estado de salud/CVRS en personas adultas mayores en condición de riesgo de abandono social en Reino Unido entre el 2007 y el 2008 en el que se utilizó un diseño cuantitativo transversal. En la investigación participaron 393 personas adultas mayores en condición de riesgo de abandono, quienes completaron un cuestionario que incluían preguntas sobre la CVRS, depresión, estado de salud, abandono social, comorbilidades físicas e información sociodemográfica. Los resultados indican que los valores de estado de salud fueron más bajos que los valores de referencia para ese grupo de edad en la población general del Reino Unido. Después de controlar edad, género, depresión, empleo, acomodaciones, comorbilidades físicas, y estado marital "viviendo solo", se obtuvo que abandono social fue significativamente asociado al estado de salud (todos las variables con $p<0.001$ y $R^{2}$ entre 0.34 y 0.41 ).

Los autores concluyen que la condición de abandono social influye en la salud y la calidad de vida de las personas adultas mayores. Se debe considerar la ganancia potencial en la CVRS de las personas en riesgo social que se obtendría si se previene y trata el abandono social.

Con respecto a la lectura crítica de este artículo, se encontró que el estudio se basa en una pregunta de investigación que es bien definida. La metodología empleada garantiza una regular validez interna del estudio. Los resultados del estudio están bien descritos. Las conclusiones presentadas están bien basadas en los resultados obtenidos y tienen en cuenta las posibles limitaciones del estudio. No hay información acerca de los conflictos de interés por parte de los investigadores. Los resultados del estudio pueden ser bien generalizables a la población y al contexto que interesa; por tanto, se considera que la calidad de la evidencia es moderada. Sin embargo, hay que considerar que la información presentada en el artículo proviene de uno más extenso, por lo que información adicional de esta investigación ha sido incluida en otras publicaciones.

Leey.Y., Chan M. \& Mok. E. (2010) Effectiveness of music intervention on the quality of life of older people. Journal of Advanced Nursing 66(12), 2677-2687. doi: 10.1111/j.1365-2648.2010.05445.x

El objetivo de este trabajo fue examinar el efecto de la música en la calidad de vida de personas adultas mayores, partiendo de la hipótesis de que después de escuchar música, las personas adultas mayores en el grupo de intervención podrían tener una mejora en la calidad de vida comparado con el grupo control.

La investigación se realizó en el 2007 y utilizó un diseño de ensayo clínico controlado aleatorizado; participaron 66 personas adultas mayores ( 65 a 90 años de edad) que fueron separadas aleatoriamente en un grupo control $(n=31)$ y en un grupo de intervención $(n=35)$. El grupo intervención fue expuesto a sesiones de 30 minutos de música por cuatro semanas, mientras que el grupo control descansaba. Los participantes completaron una evaluación inicial, antes de la separación por grupos, utilizando un cuestionario con preguntas sobre información sociodemográfica, gustos musicales, y calidad de vida. La evaluación sobre la calidad de vida fue repetida semanalmente desde la semana 1 hasta la 4 . Los resultados sugieren que la calidad de vida incrementó semanalmente en el grupo de intervención, por un efecto acumulativo de la dosis, además de que hubo una mejoría significativamente estadística en la calidad de vida del grupo de intervención comparado con el grupo control. Por ejemplo, en la semana 4, se detectó diferencias significativas entre los dos grupos en funcionamiento físico $(p=0.001)$, limitación en las actividades diarias debido a problemas físicos $(p=0.002)$, dolor corporal $(p=0.004)$, salud general $(p=0.001)$, vitalidad $(p=0.001)$, funcionamiento social $(p=0.004)$, limitación en las 
actividades diarias debido a problemas emocionales $(p=0.005)$, y salud mental $(p=0.007)$. Sin embargo, en el grupo de intervención no hubo mejoras estadísticamente significativas en las variables de calidad de vida a lo largo de las 4 semanas.

Los autores concluyen que participar en actividades musicales puede ayudar a la persona a conectarse con sus experiencias de vida y con otros individuos, y a sentirse más estimulada. La música es un método terapéutico simple, no invasivo, no costoso, y que ayuda a mejorar la calidad de vida de las personas adultas mayores que viven en la comunidad.

En relación con la lectura crítica de este artículo, se encontró que el estudio se basa en una pregunta de investigación que es bien definida. La metodología empleada garantiza una regular validez interna del estudio. Los resultados del estudio están bien descritos. Las conclusiones presentadas están bien basadas en los resultados obtenidos y tienen en cuenta las posibles limitaciones del estudio. No hay información acerca de los conflictos de interés por parte de los investigadores. Los resultados del estudio pueden ser bien generalizables a la población y al contexto que interesa; por consiguiente, la calidad de la evidencia es moderada.

Matlabi, H., Parker, S. \& McKee, K. (2011). The contribution of home-based technology to older people's quality of life in extra care housing. BMC Geriatrics, 11(1), 68-68. doi:10.1186/1471-2318-11-68

Los propósitos de esta investigación consistieron en examinar a) el uso de dispositivos electrónicos en el hogar y b) la correlación entre el uso de tales dispositivos y la calidad de vida entre personas adultas mayores que vivían en una residencia de tipo pensión (extra-care housing).

El trabajo fue en el año 2009 y utilizó un diseño cuantitativo transversal, en el que participaron 160 personas adultas mayores, reclutadas de 23 complejos residenciales en Inglaterra. Los participantes completaron un cuestionario que incluyó preguntas sobre su bienestar, calidad de vida y el uso de dispositivos electrónicos. Los resultados indican que aunque la mayoría de los electrodomésticos básicos y sistemas de llamada de emergencia son los dispositivos electrónicos más utilizados por los participantes, los otros dispositivos comunitarios ofrecidos por los complejos residenciales (e.g., lavadoras, computadoras, y equipos para el baño asistido) no son frecuentemente utilizados. Los análisis de regresión múltiple fueron ajustados para incluir las variables de confusión: edad, sexo, estado civil, nivel de movilidad, y tipo de vivienda. En este caso, el uso de los dispositivos electrónicos fue significativamente asociado con bienestar ( $b=1.17, p=0.04,95 \%$ CI [0.05, 2.29]), y calidad de vida $(b=2.83, p=0.001,95 \%$ CI $[1.17,4.50])$.

Los autores concluyen que los hallazgos del estudio deben ser considerados por las personas que están desarrollando nuevas y especializadas residencias para personas adultas mayores con limitaciones funcionales, destinando inversión en ayudas tecnológicas. Los resultados también indican que las residencias para esta población son lugares esenciales para el desarrollo y el uso de dispositivos tecnológicos.

Con respecto a la lectura crítica de este artículo en términos de la calidad de la evidencia, se encontró que el estudio se basa en una pregunta de investigación que es regularmente definida. La metodología empleada garantiza una regular validez interna del estudio. Los resultados del estudio están bien descritos. Las conclusiones presentadas están bien basadas en los resultados obtenidos y tienen en cuenta las posibles limitaciones del estudio. 
De acuerdo con la información sobre los conflictos de interés, estos no condicionan los resultados ni las conclusiones del estudio. Los resultados del estudio pueden ser bien generalizables a la población y al contexto que interesa; por tanto, la calidad de la evidencia es moderada.

Murphy K., Cooney K., Shea, E. \& Casey, D. (2009) Determinants of quality of life for older people living with a disability in the community. Journal of Advanced Nursing 65(3), 606-615 doi: 10.1111/j.13652648.2008.04929.x

Este artículo reporta los hallazgos de un estudio en el que se identificó los determinantes de la calidad de vida de personas adultas mayores discapacitadas que vivían en una comunidad, y se construyó un modelo para explicar dichos determinantes. Entre el 2005 y 2006, los investigadores utilizaron un enfoque cualitativo con un diseño de teoría fundamentada para entrevistar a 122 personas adultas mayores con diferentes discapacidades (e.g., déficit visual y auditivo y accidente vascular cerebral). A partir de los resultados, los autores desarrollaron un modelo de factores determinantes de la calidad de vida de las personas mayores con una discapacidad, el cual consta de una categoría central denominada "vivir bien", ya que era el resultado que podría esperarse cuando la calidad de vida es buena. El potencial de "vivir bien" es influido por factores basales, mediadores, y facilitadores o limitantes que incluyen la conectividad social y la respuesta adaptativa. Los resultados indican que la calidad de vida de estas personas es una compleja mezcla de estos factores.

Los autores concluyen que la calidad de vida no puede ser entendida completamente a menos que se considere la totalidad de los factores que tienen un impacto en la calidad de vida y que definen la percepción sobre esta. El modelo implica que un buen apoyo por parte del personal de enfermería, un enfoque centrado en las habilidades de la persona y acceso a información, así como la conectividad con otras personas podría hacer una diferencia y ayudar a la persona a sobrellevar la vida de una mejor manera.

La lectura crítica de este artículo mostró que el propósito del estudio fue bien definido. La revisión de la literatura $s i ́$ incluyo literatura relevante para el estudio. Con respecto al diseño del estudio, este $s i ́$ fue apropiado y sí fue basado en una perspectiva teorética, además de que el método sí fue congruente con las bases filosóficas y el propósito del estudio, lo cual se repitió con las estrategias de reclutamiento, las cuales sí fueron llevadas a cabo hasta que la saturación de la información fue alcanzada. Sin embargo, el artículo no ofrece una clara descripción del lugar donde se realizó el estudio, los participantes, el rol del investigador, y la relación con los participantes, además de que las asunciones del investigador no fueron identificadas. Aunque no hay información adecuada sobre los procedimientos para la recolección de información, los métodos para el análisis de los datos sí fueron apropiadamente descritos y poseen una naturaleza inductiva. El artículo no describe suficientemente las decisiones del investigador ligadas al análisis de la información. Finalmente, los conceptos estudiados y sus relaciones sí fueron clarificados y refinados.

Rigby, J., Payne, S. \& Froggatt, K. (2010). Review: What evidence is there about the specific environmental needs of older people who are near the end of life and are cared for in hospices or similar institutions? A literature review. Palliative Medicine, 24(3), 268-285. doi:10.1177/0269216309350253

Una revisión narrativa de literatura fue llevada a cabo para identificar y analizar evidencia sobre el tipo de ambiente físico que es necesario y preferido por pacientes mayores de 65 años de edad con un prognosis de un 
año o menos, que están recibiendo cuidado en hospitales, albergues y hogares para personas adultas mayores. Igualmente, el estudio también indagó sobre el tipo de ambiente físico necesario y preferido por los familiares y personal que labora en los lugares donde se brinda cuidado de enfermería a la población mencionada. Se incluyó artículos en inglés desde enero de 1997 : inicialmente se recuperó 575 manuscritos, de los cuales 29 fueron finalmente retenidos para el análisis ya que examinaban el ambiente físico en el contexto de temas amplios como calidad del cuidado, la experiencia de los pacientes, el personal y la familia en determinados centros de atención, y puntos de vista de los pacientes en conceptos particulares, por ejemplo dignidad o "una buena muerte". Los resultados indican que los pacientes parecen tener perspectivas más amplias acerca de su ambiente físico; no obstante, se reportó algunas diferencias entre los puntos de vista de los pacientes, sus familiares, y el personal de los centros de salud. Del análisis emergieron cuatro temas : el ambiente físico debería ser hogareño y brindar apoyo a las necesidades de interacción social y privacidad de los pacientes, además de apoyar las actividades de cuido del personal, la familia, y los pacientes y debe oportunidades para la expresión espiritual.

Los autores concluyen que el ambiente físico contribuye significativamente a la calidad de vida de las personas adultas mayores con enfermedades terminales, además de que existe una necesidad de investigación en esta área. La evaluación frecuente de las necesidades ambientales de los pacientes debe ser parte del plan de enfermería.

Con respecto a la lectura crítica de este artículo, se encontró que el estudio se basa en una pregunta de investigación que es bien definida. La metodología empleada garantiza una buena validez interna del estudio. Los resultados del estudio están bien descritos. Las conclusiones presentadas están bien basadas en los resultados obtenidos y tienen en cuenta las posibles limitaciones del estudio. De acuerdo con la información sobre los conflictos de interés, estos no condicionan los resultados ni las conclusiones del estudio. Los resultados del estudio pueden ser moderadamente generalizables a la población y al contexto que interesa. Dado lo anterior, se considera que la calidad de la evidencia es alta. Sin embargo, hay que tomar en cuenta que la mayoría de los artículos incluidos en la revisión son de naturaleza cualitativa, y la información presentada en el artículo proviene de una revisión narrativa de la literatura y no de una revisión sistemática, situación que es reconocida por los autores. Además, hay que considerar que las personas mayores no son una población homogénea, y las necesidades de las personas mayores pueden cambiar a medida que aumente la edad; por tanto, es posible que las necesidades ambientales de una persona de 70 años de edad pueden ser diferentes de las de una de 90 años de edad o de una persona moribunda, del mismo modo, las necesidades ambientales de las personas puedan variar de acuerdo con la clase social, el género, la nacionalidad, y otros factores.

\section{Nivel de recomendación de la evidencia}

Debido a la heterogeneidad en la evidencia provista por los artículos recuperados para este estudio, se ofrece dos recomendaciones basadas en dicha evidencia: la primera se refiere al impacto de la musicoterapia en la calidad de vida de las personas adultas mayores y la segunda, al impacto de múltiples factores sobre la calidad de vida de dicho grupo etario.

Respecto del efecto de la musicoterapia en la calidad de vida de las personas adultas mayores, de acuerdo con el Canadian Task Force on Preventive Health Care (2014), el grado de evidencia es I, ya que existe insuficiente evidencia (en cantidad y en calidad) para hacer una recomendación. Sin embargo, otros factores 
podrían influir la decisión, por ejemplo hay que tomar en cuenta que la información presentada por Leey et al. (2010) sugiere que las personas adultas mayores pueden participar exitosamente en actividades musicales a través de la escucha y que la música de relajación puede ser una manera de mantener un sentido de bienestar. Es evidente que intervenciones musicales afectan positivamente la calidad de vida y son un método que se puede aplicar en diferentes escenarios.

En lo respecta a la influencia de múltiples factores en la calidad de vida de las personas adultas mayores, según el Canadian Task Force on Preventive Health Care (2014), el grado de evidencia es I, ya que existe insuficiente evidencia (en cantidad y en calidad) para hacer una recomendación. Sin embargo, otros factores podrían afectar la decisión, por ejemplo, Rigby y colaboradores (2011) señalan que múltiples condiciones pueden jugar un rol en la calidad de vida de estas personas. Específicamente, la evidencia sugiere que en este grupo etario la calidad de vida recibe la influencia de múltiples factores individuales, sociales, culturales, y ambientales (e.g., apoyo social, estado de salud física, y acceso a dispositivos electrónicos).

\section{DISCUSIÓN}

Los resultados sugieren que las personas adultas mayores que frecuentemente escuchan música pueden experimentar mejoras en su calidad de vida, lo cual se evidencia al reportar una mejora en su percepción sobre su vitalidad, funcionamiento físico, dolor corporal, funcionamiento social, salud mental y general, y limitación en las actividades diarias debido a problemas físicos y emocionales, luego de participar en un programa de intervención musical, lo cual puede explicarse por el efecto de la musicoterapia en el estado de ánimo de las personas, la conciencia social, espiritual y física que facilitan la comunicación y la interacción social con otros individuos, mientras disminuyen la ansiedad y mejoran la autoeficacia y la capacidad de realizar actividades físicas que, al final, influyen en la autopercepción de la calidad de vida.

Los resultados de esta investigación son similares a los reportados por Kwon, Kim, and Park (2006), quienes investigaron los efectos de la intervención musical en el dolor, la incomodidad, y depresión en 40 adultos, mediante un diseño de ensayo clínico aleatorizado. El grupo de intervención fue expuesto diariamente a musicoterapia durante 30-60 minutos y los resultados evidencian que esta fue efectiva para reducir el dolor $(p<$ $0.001)$, la incomodidad $(p<0.001)$, y la depresión $(p<0.001)$, así como también, significativamente disminuyó la presión arterial $(p<0.001)$, la frecuencia respiratoria $(p<0.001)$ y la cardiaca $(p<0.001)$.

Con respecto al rol de diferentes factores en la calidad de vida de las personas adultas mayores, los resultados sugieren que recibe la influencia de múltiples factores individuales, sociales, culturales, y ambientales, lo cual puede explicarse por la compleja y dinámica naturaleza de la calidad de vida de este grupo poblacional que no es estática, sino que cambia constantemente debido a la exposición de la persona a diferentes experiencias, retos, y personas durante el día. Dichos resultados de alguna forma coinciden con los hallazgos del estudio descriptivo cualitativo reportados por Grewal, Lewis, Flynn, Brown, Bond, y Coast (2006) en el que 40 personas adultas mayores que vivían en una residencia fueron cuestionadas sobre su percepción acerca de la calidad de vida. Los autores encontraron que los participantes identificaron que algunos factores contribuyen a mejorar la calidad de vida, entre los que incluyen actividades, el hogar, la familia, otras relaciones, y salud entre otros. Además, los participantes reconocieron la relación entre calidad de vida y apego social, seguridad, y placer. 
La evidencia encontrada en esta investigación sugiere que la musicoterapia puede tener un efecto positivo en la calidad de vida de las personas adultas mayores. Asimismo, puede recibir la influencia positiva por otros factores, como el uso de dispositivos electrónicos, la conectividad social y la interacción positiva con otras personas; por lo tanto, la práctica de enfermería basada en la evidencia permite generar conocimientos, mejorar la educación y la práctica profesional y utilizar efectivamente los recursos disponibles, para beneficiar el objeto de estudio: el cuidado de los individuos y colectivos humanos y la calidad de vida.

Los hallazgos presentados en el presente artículo deben ser interpretados cuidadosamente ya que este estudio pose ciertas limitaciones, las cuales son discutidas a continuación. En primer lugar, todos los artículos incluidos en el análisis fueron escritos en inglés y reportan estudios llevados a cabo en Europa y Asia, por lo que la contextualización es limitada. Similarmente, los artículos reportan investigaciones en las que se reclutó diversas poblaciones de personas adultas mayores, incluyendo personas con diagnósticos de depresión y discapacidades o que vivían en diferentes modalidades residenciales, por tanto, la interpretación de estos trabajos no puede generalizarse a todas las personas adultas mayores. Además, aunque en todos los artículos el concepto de calidad de vida fue el eje central, incluyeron diferentes aspectos (por ejemplo, en el trabajo de Hawton et al. (2011), el foco de estudio fue la calidad de vida ligada a la salud), lo cual implica que la interpretación de los resultados no debe generalizarse a todos los aspectos que conlleva el estudio de la calidad de vida.

\section{CONCLUSIÓN}

Se deben llevar a cabo más estudios sobre los factores que intervienen en la calidad de vida de las personas adultas mayores, a través del metaanálisis o de revisiones sistemáticas, que permitirían corregir el vacío de información en este campo. En cuanto a estudios primarios, deberían enfocarse en la población hispana de personas adultas mayores, especialmente los costarricenses. De igual forma, las investigaciones futuras podrían considerar otros factores psicosociales como prácticas religiosas, políticas públicas, relación familiar, acceso a grupos y servicios en la comunidad.

Estos resultados pueden ser usados para complementar investigaciones futuras enfocadas en el estudio de la calidad de vida de las personas adultas mayores y mejorar la atención de este grupo de la población. Igualmente, este estudio puede ser utilizado para justificar cambios en las políticas de salud orientadas a optimizar la calidad de vida de este grupo etario. Finalmente, la construcción del conocimiento enfermero puede enriquecerse al utilizar dicha información como parte de modelos o teorías que buscan teorizar el cuidado de enfermería enfocado en la calidad de vida de las personas adultas mayores.

\section{REFERENCIAS BIBLIOGRÁFICAS}

Canadian Task Force on Preventive Health Care. (2014). Procedure Manual. Tomado de http://canadiantaskforce.ca/methods/procedural-manual/\#fn-2

Chan, S., Shoumei, J., Thompson, D., Yan, H., Chiu, H., Chien, W. \& Lam, L. (2006). A cross-sectional study on the health related quality of life of depressed Chinese older people in Shanghai. International Journal of Geriatric Psychiatry, 21(9), 883-889. doi:10.1002/gps. 1578 
Castrillón, M. (2001). Teoría y práctica de la enfermería: los retos actuales. Investigación y Educación en Enfermería. Universidad de Antioquia. XIX(1), 55-56

Galváo, C., Sawada, N. y Mendes, I. (2003). A busca das melhores evidencias. Revista Ese. Enfermagem.USR 37(4), 43-50

Gómez de la Cámara, A. (Ed.). (1998). Manual de Medicina Basada en la Evidencia. Elementos para su Desarrollo y Aplicación en Atención Primaria. España: Editorial: Jarpyo.

Grewal, I., Lewis, J., Flynn, T., Brown, J., Bond, J. y Coast, J. (2006). Developing attributes for a generic quality of life measure for older people: preferences or capabilities? Social Science \& Medicine 62, 1891-1901.

Hawton, A., Green, C., Dickens, A., Richards, S., Taylor, R., Edwards, R., Greaves, C. \& Campbell, J. (2011). The impact of social isolation on the health status and health-related quality of life of older people. Qual Life Res, 20:57-67. Doi 10.1007/s11136-010-9717-2

Icart, T. (2000). Enfermería basada en la evidencia. Ponencia presentada en la II Mesa redonda: Gestión de los cuidados. II Congreso de Enfermería Comunitaria, Molina, Málaga. Recuperado de http://www.asanec.org/Congreso/EBE.htm

Kwon, I., Kim, J. \& Park K. (2006) Effects of music therapy on pain, discomfort, and depression for patients with leg fracture. Taechan Kanho Hakhoe Chi 36(4), 630-636.

Leey.Y., Chan M. \& Mok. E. (2010) Effectiveness of music intervention on the quality of life of older people. Journal of Advanced Nursing 66(12), 2677-2687. doi: 10.1111/j.1365-2648.2010.05445.x

Letts, L., Wilkins, S., Law, M., Stewart, D., Bosch, J., \& Westmorland, M., (2007). Guidelines for Critical Review Form: Qualitative Studies (Version 2.0). Tomado de http://www.srs-mcmaster.ca/Portals/20/pdf/ebp/qualreview_version2.0.pdf)

Lopes, D., de Mattos, C. (2005). Prática basada em evidencias, aplicada ao raciocinio diagnóstico. Revista Latino-americana de Enfermagem 13(3), 415-422.

López M., Reviriego, E., Andrío, E., Rico, R., Sobradillo, N., Hurtado, I. (2006). Revisión externa y validación de instrumentos metodológicos para la Lectura Crítica y la síntesis de la evidencia científica. Madrid: Plan Nacional para el SNS del MSC. Servicio de Evaluación de Tecnologías Sanitarias del País Vasco (Osteba). Informes de Evaluación de Tecnologías Sanitarias: OSTEBA No 2006/02.

Matlabi, H., Parker, S. G., \& McKee, K. (2011). The contribution of home-based technology to older people's quality of life in extra care housing. BMC Geriatrics, 11(1), 68-68. Doi:10.1186/1471-2318-11-68

McKibbon, K. (1998). Evidence-based practice. Bull Med Libr Assoc, 86(3):396-401

Murphy K., Cooney K., A., Shea E. \& Casey, D. (2009). Determinants of quality of life for older people living with a disability in the community. Journal of Advanced Nursing 65(3), 606-615. Doi: 10.1111/j.1365-2648.2008.04929.x

Ochoa, S. (2005). A propósito de la enfermería basada en la evidencia: algunos cuestionamientos, limitaciones y recomendaciones para su implementación. Investigación y Educación en Enfermería, XXIII Septiembre, 138-146 


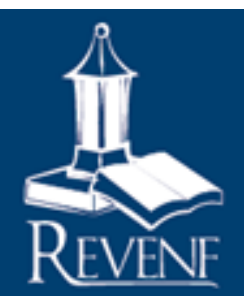

\section{Revista Electrónica Enfermería Actual en costa Rica}

Rigby, J., Payne, S. \& Froggatt, K. (2010). Review: What evidence is there about the specific environmental needs of older people who are near the end of life and are cared for in hospices or similar institutions? A literature review. Palliative Medicine, 24(3), 268-285. doi:10.1177/0269216309350253

Rojas, L., Cubero, C., y Leiva, V. (2014). Investigación secundaria: utilización de la mejor evidencia en la práctica clínica. San José: CIEBE, UCR.

Urra, E., Retamal, C.; Tapia, C., \& Rodríguez, M. (2010). Enfermería basada en la evidencia: qué es, sus características y dilemas. Investigación y Educación en Enfermería, 28(1), 108-118.

WHOQOL GROUP (1994). The IQOLA Proyect Group. The SF-36 health survey: Development and use in mental health research and the IQOLA Proyect. International Journal of Mental Health, 23, 49-73. 